\title{
Adaptive Algorithm Recommendation and Application of Learning Resources in English Fragmented Reading
}

\author{
Jinyu Cheng ${ }^{1}$ and Hong Wang $\mathbb{D}^{2}$ \\ ${ }^{1}$ International Education College, Wuhan Sports University, Wuhan 430079, China \\ ${ }^{2}$ Wushu College, Wuhan Sports University, Wuhan 430079, China \\ Correspondence should be addressed to Hong Wang; 2004071@whsu.edu.cn
}

Received 5 February 2021; Revised 8 March 2021; Accepted 17 March 2021; Published 30 March 2021

Academic Editor: Wei Wang

Copyright (c) 2021 Jinyu Cheng and Hong Wang. This is an open access article distributed under the Creative Commons Attribution License, which permits unrestricted use, distribution, and reproduction in any medium, provided the original work is properly cited.

\begin{abstract}
This paper firstly designs a five-dimensional model of learners' characteristics (learners' English reading ability, cognitive style, learning goal, learning situation, and learning effect) and a three-dimensional model of English reading resources' characteristics (question types, topics, and difficulty of resources) in a fragmented learning environment through literature research. At the same time, to make the learning resources meet the characteristics of fragmented learning time and space, the English Level 4 reading resources are reasonably designed and segmented to adapt to the needs of learners' mobile fragmented learning. Then, combined with machine learning algorithms, an adaptive recommendation model of learning resources in English fragmented reading is constructed. The algorithm-based adaptive recommendation algorithm for English fragmented reading resources is designed. Based on the generated decision trees, the expression rules are parsed to achieve adaptive pushing of resources. The results of this study show that adaptive recommendation of learning resources in English fragmented reading can help teachers to develop future resource recommendation strategies through effective data collection to adaptively push resources that are close to learners' individual needs. The use of mobile by English learners to learn to read in a fragmented learning context enables targeted training in weak areas of English reading, thus enhancing different aspects of learners' reading skills.
\end{abstract}

\section{Introduction}

With the rapid development of information technology, people can use handheld mobile devices such as cell phones and PDAs to obtain, process, and send information at any time or place, making communication and information ubiquitous and providing us with the possibility to rely on handheld mobile devices and wireless networks to carry out educational activities and deliver educational information for lifelong human learning [1]. In this era of popular handheld mobile devices, especially smartphones and tablets, college students almost have one in their hands. In this environment where learning can be done anytime and anywhere, people's learning habits and behaviors are quietly changing, and fragmented learning has become one of our main learning styles. The application of fragmented learning in the field of education is gradually gaining attention, and its application in language subjects is more frequent, especially in English learning. With the rapid development of information technology, fragmented learning is also the most responsive strategy to the changes in English learning and aids beyond the systematic learning of English [2]. Fragmented reading is also becoming increasingly popular among college students, and traditional paper-based reading methods are difficult to meet the needs of young people. With the many course tasks and academic pressure that college students are taking, using this new style of reading allows students to use their fragmented time to learn and accumulate English knowledge, rather than being limited to the limited time and space of the English classroom [3]. Students can use fragmented learning to learn English independently according to their needs and receptive abilities to increase their interest and learning effectiveness in English. Since the two most fundamental characteristics of 
fragmented learning are the discrete and fragmented learning time and learning space, fragmented learning naturally lends itself to the small granularity of learning, such as vocabulary and grammar [4].

Adaptive learning systems are different from traditional e-learning in that traditional e-learning systems do not pay attention to individual differences of learners and only provide the same learning resources and strategies, which can easily cause cognitive overload and network disorientation of learners, while adaptive learning systems dynamically present appropriate learning resources and learning activities based on learner characteristics and learning needs in the process of mobile fragmented learning. In contrast, adaptive learning systems present appropriate learning resources and learning activities based on learners' characteristics and learning needs in the process of mobile fragmented learning, thus stimulating learners' motivation, cultivating learning autonomy, and improving learning efficiency [5]. Although current adaptive learning systems have personalized features, many of them are mainly based on the judgment of learners' current knowledge level, and then, filter the learning resources suitable for the current level, without fully considering the different needs, learning preferences, and learning contexts of learners [31]. Therefore, in the research of adaptive learning systems, the key lies in the establishment of learner models and the knowledge recommendation and service customization based on the established learner models. However, in the past, a single method was used to construct learner models, which often ignored the variability of feature terms and easily resulted in unsatisfactory recommendation effects [6].

In this paper, we collect and record learners' data in the learning process based on their personalized needs in a mobile and fragmented learning environment, combine them with reading resource feature data, and make them suitable for data mining through preprocessing. The ID3 algorithm is then used to build a decision tree for resource pushing, from which resource pushing rules are identified, to achieve personalized pushing of English Level 4 reading resources in the mobile learning environment and to make the weak links in learning get targeted reinforcement training to meet the personalized learning needs of learners. In the fragmented learning environment, it is necessary to recommend learning resources to learners based on users' personality characteristics to meet learners' personalized learning needs. The thesis tries to first study English fragmented reading and adaptive learning system, and in the process of the study, we establish five-dimensional characteristic information based on learners' reading ability, cognitive style, learning goal, learning situation, and learning effect in the process of English fragmented reading. We also extract the three-dimensional feature information of English reading resources, establish the connection between the learner model and learning resources, and use this as the basis to determine the resource pushing strategy and build the adaptive recommendation model of learning resources in English fragmented reading.

\section{English Fragmented Reading Resources' Adaptive Recommendation Application}

2.1. Related Work. Jelonek and Mazur developed an adaptive learning system for spatial geometry that can match learners' characteristics and learning resources based on the similarity between them and push relevant learning resources based on the diagnosis of learners' learning [7]. Rodrigues et al. developed an adaptive learning system for learning sciencebased knowledge for 12 to 14 year olds [8]. Sreeram and Vuppala designed an adaptive learning system using fuzzy item reflection theory, which can diagnose students' learning and push relevant learning resources through students' feedback [9]. Mohammadi et al. designed an adaptive learning system that structured the course, represented the course resources as ontologies, diagnosed the students' learning based on the ontology structure and ontology reasoning mechanism, recommended different sequential learning resources for the students, and determined the students' usage time based on the diagnosis results [10]. Wei et al. conducted relevant research on personalized recommendation systems and found that personalized recommendation systems have strong adaptiveness to learners and the Internet and can achieve personalized recommendation services by analyzing learner behavior [11]. After years of development, foreign personalized recommendation systems have become increasingly diverse in terms of recommendation methods and increasingly personalized and intelligent in terms of recommendation service effects.

The Learning Lab (SSL) developed at Stanford University, USA, attempted to combine foreign language learning content with cell phones to develop foreign language learning models to exercise learners' language skills, and this pilot study showed that, in learning using cell phones, learners' attention tends to be highly fragmented and that the use of fragmented time for learning on the move raises a variety of new contextually relevant issues that are very different from others [12]. There is a significant difference between multiple learning of a large number of systems at a fixed location. Therefore, this "fragmented" learning style in mobile learning requires fragmented learning materials [13]. In the mobile environment, it is difficult to control the influence of external factors and to maintain continuous and focused attention for a long time [14]. Learning platforms related to fragmented learning have also been studied, which fully demonstrate the advantages of fragmented learning and can provide the possibility for learners to learn through fragmented learning, which better meets the needs of learners' fragmented learning and lays the foundation for this paper to learn English reading in a fragmented learning way [15].

The research on adaptive learning systems has evolved from focusing on learning content and adaptive navigation for learning content presented at the beginning to later exploring learner models in depth, while incorporating educational psychology factors such as learning styles into them and then gradually improving to personalized learning support services such as mining learners' personalized 
learning needs. The research focuses on analyzing learners' characteristics based on the needs of mobile fragmented learning resources, collecting learners' personalized characteristics data, and mapping the collected information to the attributes of resources after certain processing, to provide learners in the "learner-centered" English fragmented self-directed learning mode with personalized learning support services. The result is an adaptive recommendation system for learning resources in English fragmented reading and its application. The following four aspects will be studied: the adaptive recommendation model of learning resources in English fragmented reading, the design of English learning resources in fragmented reading, the design and implementation of the adaptive recommendation system of learning resources in English fragmented reading, and the application and evaluation of the adaptive recommendation system of learning resources in English fragmented reading.

2.2. Adaptive Recommendation Algorithm. The principle of collaborative filtering is to find similar users with the same preferences or interests as the current user according to each user's preferences for resources or items, which is also called neighboring users. Item-based collaborative filtering is the counterpart of user-based collaborative filtering, which recommends similar resources or items to the current user according to the selection history of neighboring users or similar users. The implementation principle is the same as that of the user-based collaborative filtering, which recommends similar resources or items to users according to their selection history of items. The basic idea of adaptive resource pushing is to automatically adjust the recommendation strategy according to the change of learner attributes, to adapt to the change of learners, and push the most suitable learning resources [16]. However, not every dimension in the learner model is updated at the same rate. If only some of the learner attributes are involved in each push strategy update, this can greatly reduce the computational effort, improve the system response speed, and thus improve the user experience.

In the learner model, the learner's cognitive style is the habitual behavioral pattern that the individual shows in the cognitive process, which is a process variable rather than a content variable with stability across time and consistency across contexts; therefore, it can be assumed that the individual's cognitive style is fixed for a relatively long period; the learner's English reading ability is the ability to comprehend, analysis, generalization, guessing, and judging abilities, which are adjusted over time and usually change after a longer period of study; learning goals refer to the gradual changes in learners' learning levels and skills through the study under a set plan to approach or get closer to the goals. Special emphasis is placed on the changes in knowledge, skills, and emotional behaviors that learners should achieve after completing a certain stage or unit of the study. The learning goals in English reading are mainly the number of words that learners can increase and the percentage of correct questions that they can achieve through learning in a stage. Therefore, learning goals change over some time; learning context is the physical environment in which learners are learning, which will change all the time with the change of the learning environment; the learning effect is the result produced after completing a certain unit of learning, so it will change consequently because of certain learning of learners, as shown in Figure 1.

As can be seen from the previous section, the initial classification category is judged by the "goodness of the recommended resources," which is judged by the self-rated difficulty of the learners, and when the self-rated difficulty of the learners is medium, the recommended resources are suitable; when the self-rated difficulty of the learners is hard and easy, the recommended resources are not suitable:

$$
\begin{aligned}
\operatorname{Entropy}(X) & =\operatorname{Entropy}\left(P_{1}, P_{2}\right), \\
& =-\sum_{i=1}^{n} P_{i} \log _{2}^{3} P_{i}, \\
& =-\frac{X_{\text {yes }}}{X} \log _{2} \frac{X_{\text {yes }}}{X}-\frac{X_{\text {no }}}{X} \log _{2} \frac{X_{\text {no }}}{X} .
\end{aligned}
$$

The information metric evaluation function is crucial to the MIFS-based feature selection method, and although the functions take various forms, they all aim to select the subset of features with the greatest relevance to the category [91]. The generalized information metric evaluation function can be expressed as

$$
J(f)=a \oplus g(C, f, S)^{2}-\delta^{2},
$$

where $S$ is the selected feature, $f$ is the candidate feature, $C$ is the category, the function $g(C, f, S)$ is the amount of information between $C, f$, and $S$ and the degree of correlation between the selected feature with the addition of the candidate feature and the category, $\alpha$ is the adjustment factor to adjust the degree of information brought by $f$ when adding is the degree of information brought when $f$ is added, and $\delta$ is the penalty factor for the degree of redundancy brought by $f$ to $S$. Thus, the simplest and most intuitive information metric evaluation function can be

$$
J(f)=g(C, f)^{2},
$$

where $g(C, f)$ is the mutual information and $\beta$ is the adjustment coefficient. At this point, the evaluation function is calculated directly for all candidate features to prevent excessive redundancy in the preprocessing, and the correlation between features is considered, and $f$ is penalized by the correlation between feature $f$ and the selected single feature $s$. The final evaluation function can be expressed as follows:

$$
J(f)=g(C, f)^{2}-\beta \sum_{s \in S} g(C, f)^{2} .
$$

It is especially important to determine learners' preference for learning resource features, and the selected features represent some features that determine whether they will affect learners' choice of resources, such as knowledge content of resources and learning duration. This part of features can be determined based on the combing of known 


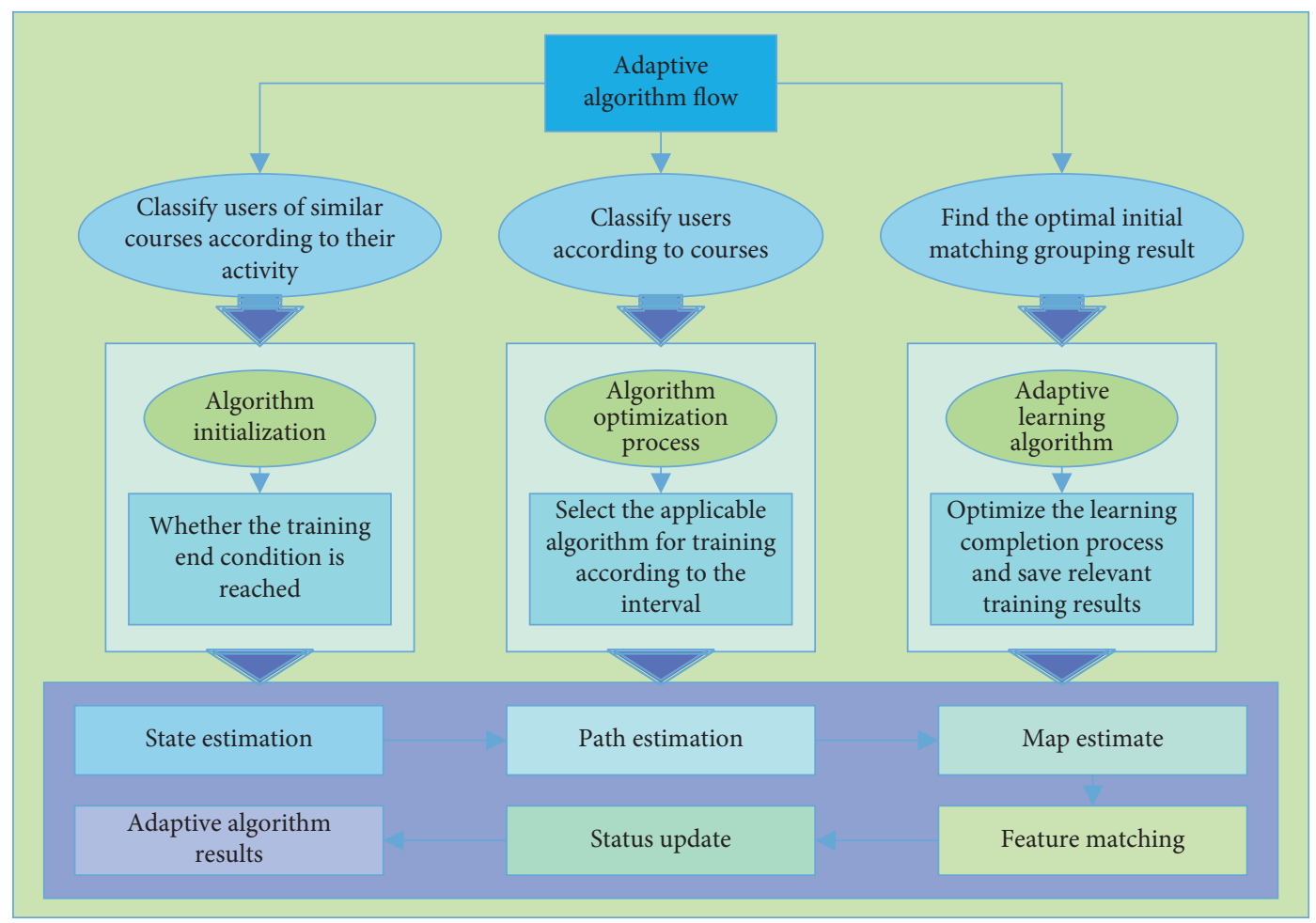

FIGURE 1: Adaptive recommendation algorithm.

studies or by survey; the candidate features represent some features that cannot be determined yet whether they affect learners' learning of a resource, such as learners' age; the category is the extraction of the selected features, which is used to measure the relevance of the information of the selected features to the candidate features [16]. The final evaluation function is constructed to add the candidate features with greater relevance, judge the impact of the candidate features on the evaluation results, and then filter out some redundant features, to effectively alleviate the workload of deep learning training using the data later.

The number of times learners have studied a learning resource or certain learning resources is of great help in understanding learners' behavior and interests. Traditional collaborative filtering recommendation algorithms mainly rely on whether learners have learned certain learning resources and on learners' preferences, lacking consideration of the number of times different learners have learned the resources and simply assuming that learners are interested in the resources as long as they have learned them. Usually, the number of times learners learn the same resource or the same type of resource represents different levels of preference, so the number of times learners learn the resource is a good indicator of learners' preference. Such a simple normalization does not objectively reflect how much learners care about a learning resource, so the frequency of learning a particular resource cannot be ignored:

$$
X_{m \times n}=\left[\begin{array}{cccc}
X_{11} & X_{21} & \ldots & X_{n 1} \\
X_{12} & X_{22} & \ldots & X_{n 2} \\
\ldots & \ldots & \ldots & \ldots \\
X_{1 m} & X_{2 m} & \ldots & X_{n m}
\end{array}\right] .
$$

The frequency of learning resources by learners can reflect different levels of preference, and the average frequency of resource learning can be defined as

$$
\overline{r\left(l_{i}\right)}=\frac{\sum_{i=1}^{j} k\left(l_{j}, r_{i}\right)}{n^{2} r\left(l_{i}\right)} .
$$

Since the size of this frequency is highly related to whether the learner is interested in learning the resource and a learner who learns a learning resource more often can be considered to have a higher attention to that resource or that type of resource, the method in this paper also predicts the specific learning frequency, compares it with the actual number of learning, and judges its error size, i.e., the regression analysis of the recommendation model, further to decide whether certain learning is recommended to the learner or not.

\subsection{English Fragmented Reading-Learning Applications.} The concept of lifelong learning is gradually gaining popularity, and people's demand for learning is increasing, and they are eager to learn increasingly to cope with the changes 
of the times. Stimulated by this demand, many learning apps have emerged, and although most of these apps have a large number of learning resources, it seems that people can learn a lot by using the software but it is difficult for them to quickly select the part they need when they are faced with a huge amount of information. The pressure of information overload makes it difficult for people to get better improvement through learning. Therefore, this project investigates the problem of adaptive pushing of English reading resources in a fragmented learning context. Based on the learner characteristics, resource characteristics, and the influence of environmental volume on the learner during the learning process, the app analyzes the learner's learning needs based on the learning history data when the learner logs in again and adaptively pushes the resources to help the learner get the information they need quickly [17]. Therefore, the app can be divided into the following functions: login and registration, context acquisition, learning resources recommendation, independent practice, and learning process record. In addition to the functional requirements, the app should also have nonfunctional requirements and should be secure, simple to use, and appropriately scalable to accommodate the future needs of users.

The system is built to better help decision-makers develop future recommendation strategies through an effective dataset so that the weak links in learning can be targeted and reinforced through the training learning system. According to the overall architecture of English fragmented reading resources' adaptive push app development shown in Figure 2, the overall framework diagram of the system is divided into five layers, specifically including the foundation layer, data layer, service layer, application layer, and user layer. The foundation layer is the bottom layer of the system architecture, which is mainly based on the campus network (LAN) to support the network service of the adaptive learning resource recommendation system and meet the network service requirements of the system. The data layer is the second layer of the system framework, which mainly operates and manages the system data, including resource data, management data, behavior data, and evaluation data. The resource data includes English reading resources, cognitive style test resources, and English reading ability test scale resources; the management data includes students' personal information and software information; the behavior data includes learners' behavior data, self-set study plans, and semester tests; the evaluation data includes answer practice results' data. The data layer is mainly responsible for collecting, processing, exchanging, and storing the above data.

The service layer is the middle layer of the overall system architecture, which is used to connect the data layer and the application layer. The service layer is mainly used to support the implementation of the system in the application layer to provide application services to users, mainly using the ASP.NET WebAPI framework, which can quickly provide APIs for HTTP clients to create web services. The application layer presents the adaptive learning services that the adaptive learning resource recommendation system eventually provides to learners. It mainly reflects the functions of login and registration, learning resource recommendation, independent practice, context acquisition, and learning record provided by the system. Among them, log in and registration is used for identity verification and testing of reading ability and cognitive style; learning resource recommendation is mainly used for the system to recommend learning resources for learners; independent practice is mainly used for learners to select learning modules and set learning plans according to their own learning needs; context acquisition is mainly used to obtain the ambient volume of the current user's geographical location. The learning record mainly records the basic information and learning behavior of the current learners.

The learner's basic information table is shown in Table 1, which mainly records the basic information of the learner as well as the type of cognitive style test and the level of the initial reading ability test.

It is difficult for learners to find the appropriate learning resources quickly and accurately from the huge amount of resources, so the adaptive learning resource recommendation app described in this paper is mainly to solve this problem. By collecting the characteristics data of learners in the learning process and combining the three-dimensional characteristics of reading resources, we use the decision tree ID3 algorithm to make an adaptive recommendation of resources [18]. The learning record mainly includes the basic information of current learners, message reminder notification, learner behavior records (error set, reading history, my plan, and learning progress), user test records (reading ability and cognitive style), and the basic setting information of the software.

For the 200 learner knowledge-space models obtained from the simulation, use the contribution-based learning target selection algorithm and the learning cost-based learning target selection algorithm to obtain the corresponding sets of learning target selection results; for each learning target obtained in the previous step, use the domain knowledge ontology to obtain the set of knowledge points that learners of the current knowledge space model need to learn and have not yet mastered to achieve the learning target [19-22]. For the set of knowledge points obtained in the previous step, the bottom-up knowledge point-learning sequence-planning algorithm is used to obtain the knowledge point-learning sequence-planning results for learners with the sequential learning style, and the left-to-right knowledge point-learning sequence-planning algorithm is used to obtain the knowledge point-learning sequenceplanning results for learners with the comprehensive learning style.

For the learning target selected by the contributionbased learning target selection algorithm, evaluate whether the learning target has a high contribution to the learner's future mastery of relevant skills and competence in actual production jobs; for the "job" learning target selected by the learning target selection algorithm based on learning cost, combined with the set of knowledge points that learners need to learn and have not yet mastered, evaluate whether the set covers all the knowledge points that learners must 


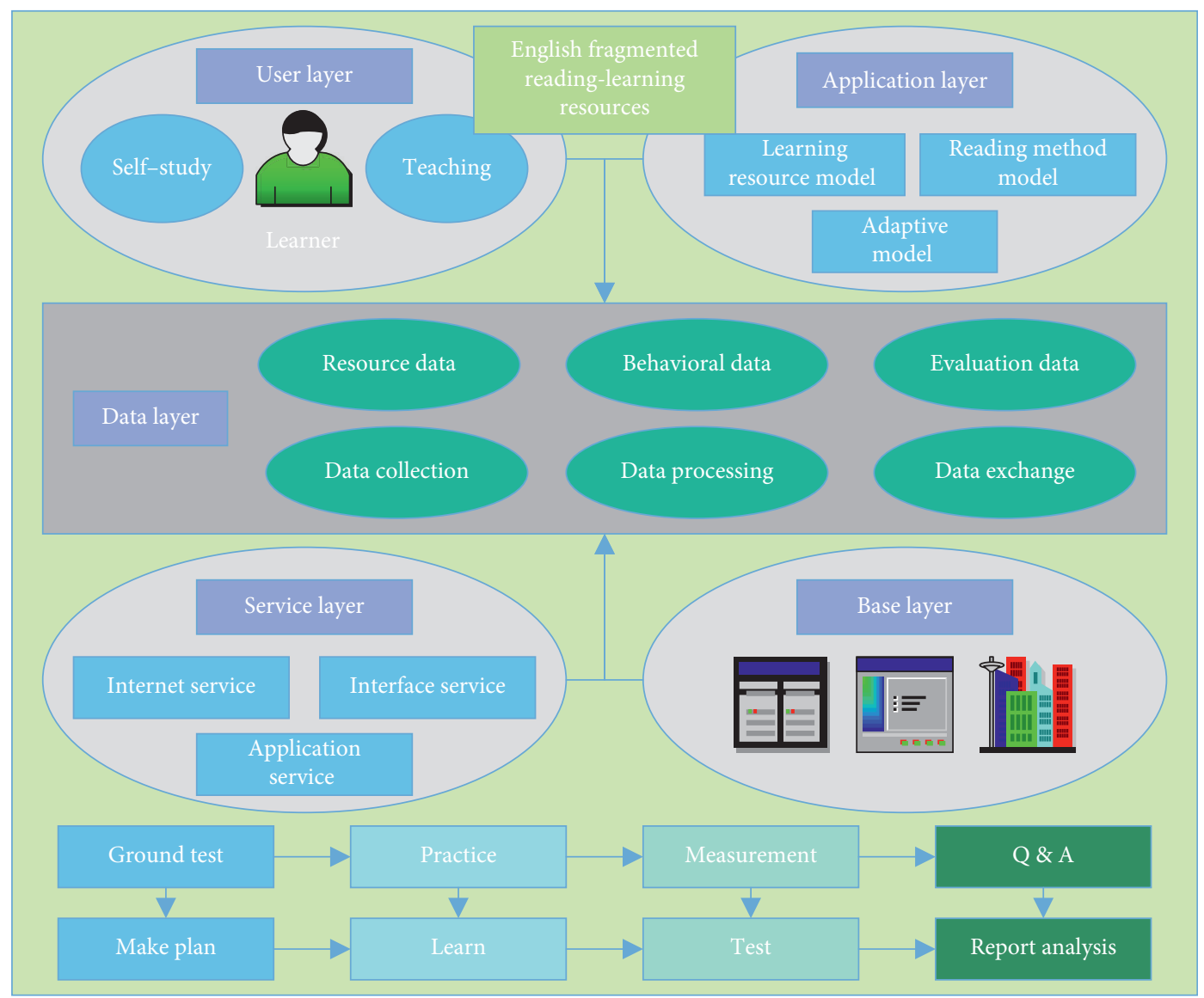

Figure 2: Overall system architecture diagram.

TABle 1: User table.

\begin{tabular}{lcc}
\hline Name & Field name & Type \\
\hline User ID & ID & String \\
Username & User name & String \\
User password & Password & String \\
User mailbox & E-mail & String \\
Registration time & Register time & String \\
Last login time & Last login & String \\
Cognitive style & Cognition & Int \\
Reading ability level & Ability & Int \\
Reading ability test time & Ability time & String \\
\hline
\end{tabular}

master to achieve the learning goal and whether it contains knowledge points that are not helpful to achieve the learning goal; based on the learning style of learners based on the learner's learning style, we evaluate whether the result of the learning order of knowledge points obtained from the planning is consistent with the learner's knowledge pointlearning order preference.

\section{Analysis of Results}

3.1. Resource Push Analysis. It is clear from the theory of the nearest neighbor zone that the most appropriate learning content for the learner should be slightly above the learner's current level. Therefore, the difficulty is an important factor considered in resource pushing and is generally set by the question writer based on experience. Whether or not the questions are done correctly directly reflects the effectiveness of learning and is the primary goal of the learning assistance system. Learner self-assessed difficulty reflects the learners' subjective perception of the learning status, quality of the resources, suitability of the learning environment, and suitability of the resource delivery algorithm and is used to test the effectiveness of the recommendations from the learners' subjective perspective. Ideally, the difficulty should be the same as the self-assessment difficulty, and both should be slightly higher than the correct error rate.

The correlation coefficient between self-rated difficulty and topic difficulty was 0.365 , and the correlation coefficient between self-rated difficulty and correct errors (actual difficulty) was 0.163 , as tested by Spearman's rank correlation coefficient, indicating a moderate correlation between learners' self-rated difficulty and the difficulty of the systematic topics and between learners' self-rated difficulty and correct errors. This indicates that the empirically set difficulty of the questions is consistent with the learners' perception of the difficulty of the questions. Moreover, whether the questions were done correctly was consistent with the learners' perception of question difficulty, as shown in Figure 3.

The correlation coefficient between the positives and errors of doing the questions and the difficulty of the system 


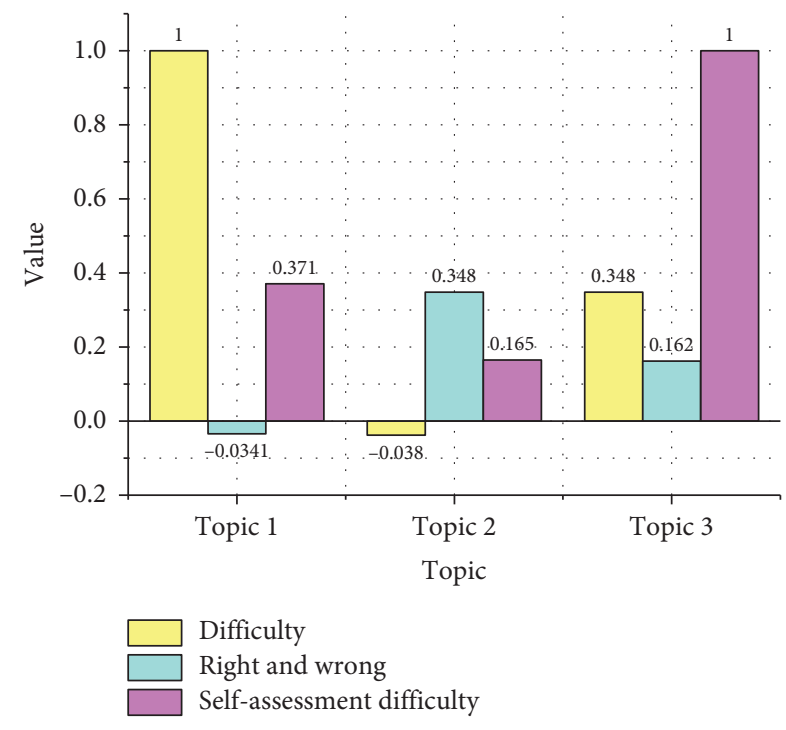

FIGURE 3: Correlation analysis of difficulty, correctness, and selfassessment.

questions was -0.0341 , indicating that the correlation between the positives and errors of doing the questions and the difficulty of the system questions was not significant. This result suggests that the empirically set question difficulty does not affect learners' correctness or error in answering questions. Instead, the system question difficulty should be revised dynamically based on the learner's learning process data so that it can be flexibly adapted to the individualized level of the learner.

The attribute values of question type, question material, and difficulty in the resource model were correlated with the correctness and self-assessed difficulty of doing the questions, as shown in Figure 4.

The correlation coefficient between the resource's question model and the positive errors of doing the questions was -0.123 at a significant level of 0.001 , as detected by the Spearman's rank correlation coefficient, indicating that the resource's question model was significantly negatively correlated with the positive errors. The resource models were numbered as (1) factual detail questions, (2) reasoning and judgment questions, (3) semantic comprehension questions, (4) main idea questions, and (5) opinion and attitude questions. It is generally believed that the larger the value of the question type, the more difficult it is, i.e., the question type is positively correlated with the positive error. However, the results obtained showed the opposite. This may be related to the ratio of the number of questions of various topics in the question bank, the learners' level of preparation when doing the questions, or how the fragmented resources are split.

The correlation analysis was done between the reading ability, cognitive style, learning goal, and volume cacophony in the learner model and the correctness and self-assessment difficulty of doing the questions, as shown in Figure 5.

The correlation coefficients of reading ability, learning objectives, and correct errors were 0.14556 and 0.18634 ,

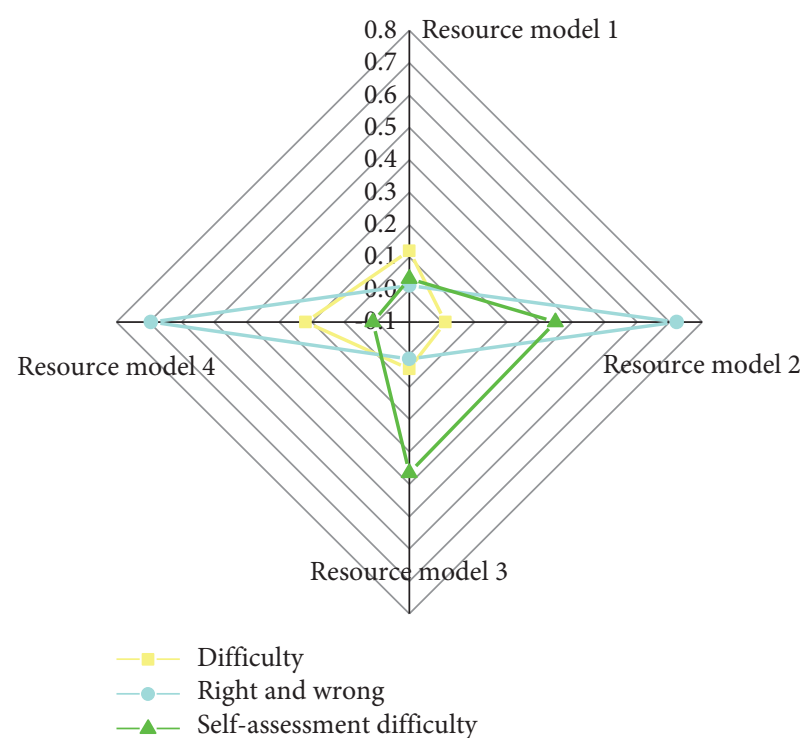

FIgURe 4: Correlation analysis of resource model and positive errors.

respectively, as tested by Spearman's rank correlation coefficient, indicating a moderate correlation between correct errors in doing the questions and the learners' reading ability and the presence or absence of set learning objectives. The reading ability is numbered as follows: (1) Level 4, (2) Level 5, (3) Level 6, and (4) Level 7. If learners with low reading ability do better in the questions, it indicates that the recommended resources are more reasonable and can improve the learning effect of learners with low reading ability, and the English reading resources involved are the real questions of college English Level 4; then, the learning effect of learners with a high level is not obvious. The learning objectives are numbered 1 and 2 . It is generally believed that learners with learning goals are highly motivated and have higher learning effects, just the opposite; thus, learning goals need to be further tested. The correlation coefficient between learners' cognitive style and positive errors was -0.0924 with a significance level of $0.011<0.05$, indicating that learners' cognitive style is significantly negatively correlated with positive errors. The cognitive styles were numbered as (1) field-dependent and (2) field-independent. This indicates that the selected learners with the field-independent style did better on the questions than the learners with the fielddependent style. However, this result is not necessarily universal and may be related to the selected learners. Moreover, the system is not perfect for obtaining the decibel value of the volume noise level, which can only be obtained by manual selection and only at one login, which may result in the pushed learning resources not being sensitive to changes in the learning environment, and thus, the effect of the volume noise level on the learning effect is not relevant.

3.2. System Application Analysis. The learning effect is mainly evaluated from the learners' correctness rate of doing the questions, but since the recommended resources in this system are recommended differently based on different 

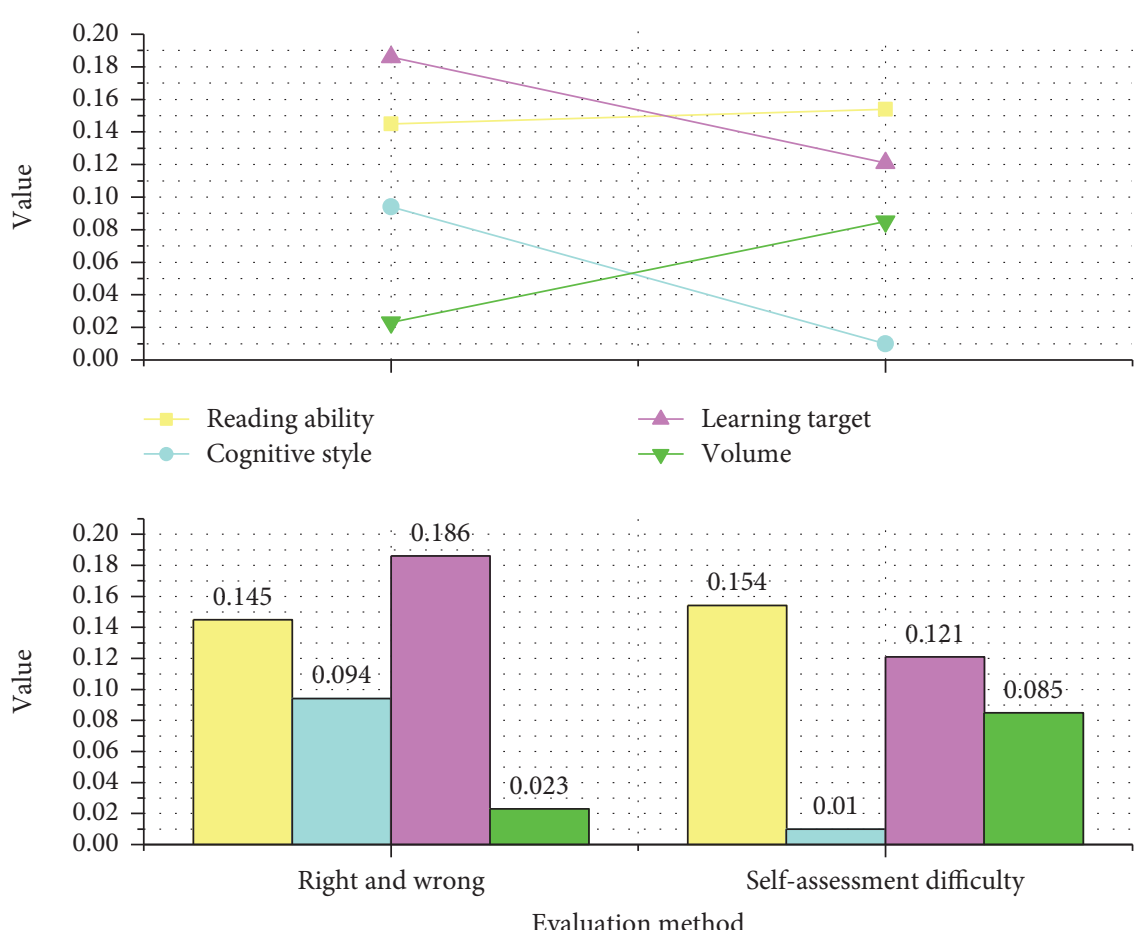

Reading ability Cognitive style

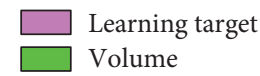

FIgURE 5: Correlation analysis of learner models and positive errors.

learning data of different learners, the length of learning and the size of the resources are different; thus, the different learning processes and different learning results of different learners cannot be evaluated uniformly with only a single evaluation. In this study, the evaluation of learning effectiveness was conducted by analyzing only those learners in the system who were able to learn continuously.

As can be seen in Figure 6, the learners were able to use the app to assist in the learning of English fragmented reading continuously, and the learning effect was generally on the rise, indicating that the use of the app to assist in the learning of the English Level 4 close-reading module could improve the learners' learning ability, and the learning effect was also increasing with time, and the correct rate of doing the questions was also increasing. Due to the short duration of the learners' study and the complex learning environment of mobile fragmentation during the learning process, which also influences more factors than just the decibel value of the volume noise level, it is obvious that the correct rate of doing the questions in the line graph also occasionally decreases.

The learners' current reading ability is Level 5, the cognitive style is field-independent, and the learning effect is relatively stable showing an upward trend, while for the field-independent learners, it is not easy to be influenced by the environment, but the rise is not great, which is related to the learners' current reading ability, the Level 5 reading ability in this study which is comparable to the level of

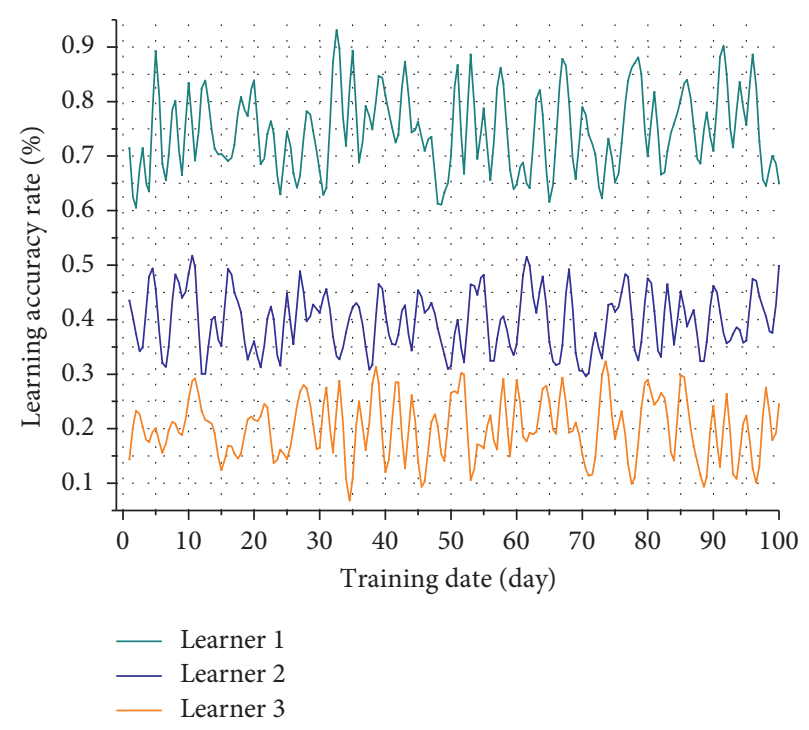

FIgURE 6: Typical learner's daily correct learning rate.

English Level 4, and the reading resources are comparable to the level of college English Level 4 reading materials equivalent, so it is difficult for learners to do Level 4 topics. The above analysis shows that, through the recommended resources of this system, learners can learn English fragmented reading for about one month, and from the analysis 


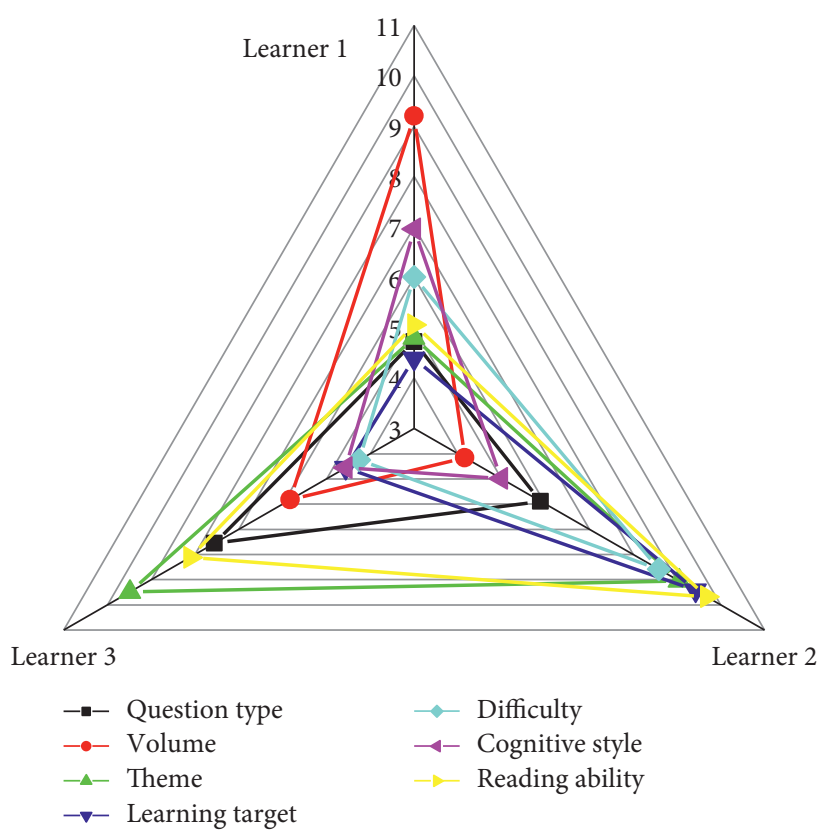

FigURE 7: Radar map of typical learner characteristics distribution.
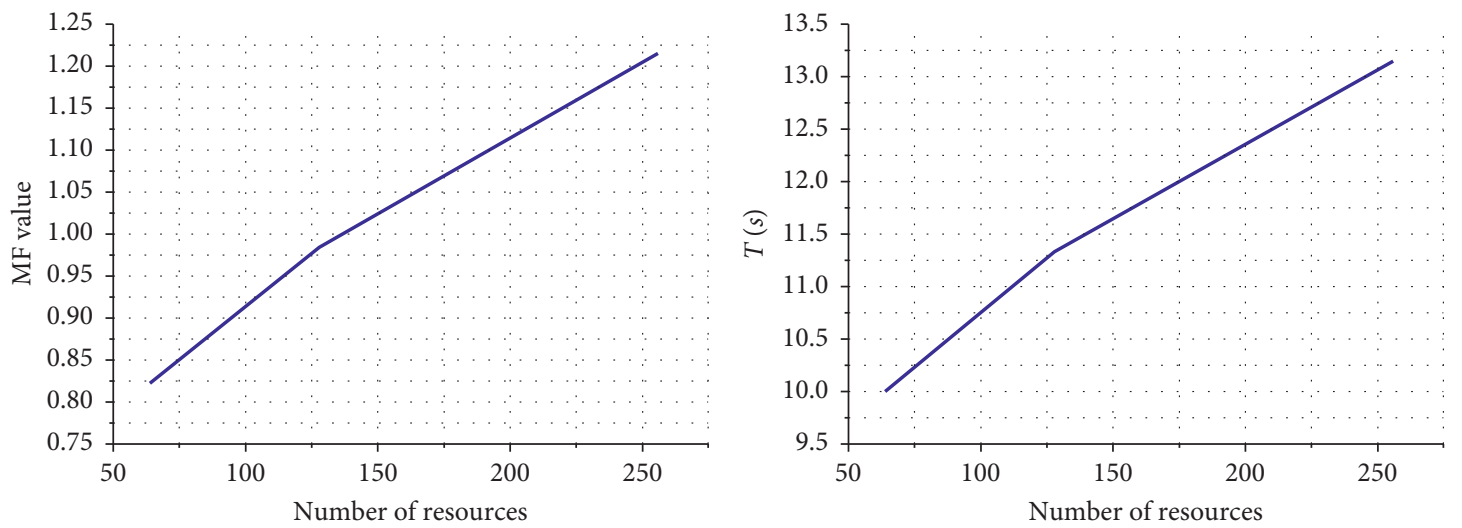

_ Genetic algorithm
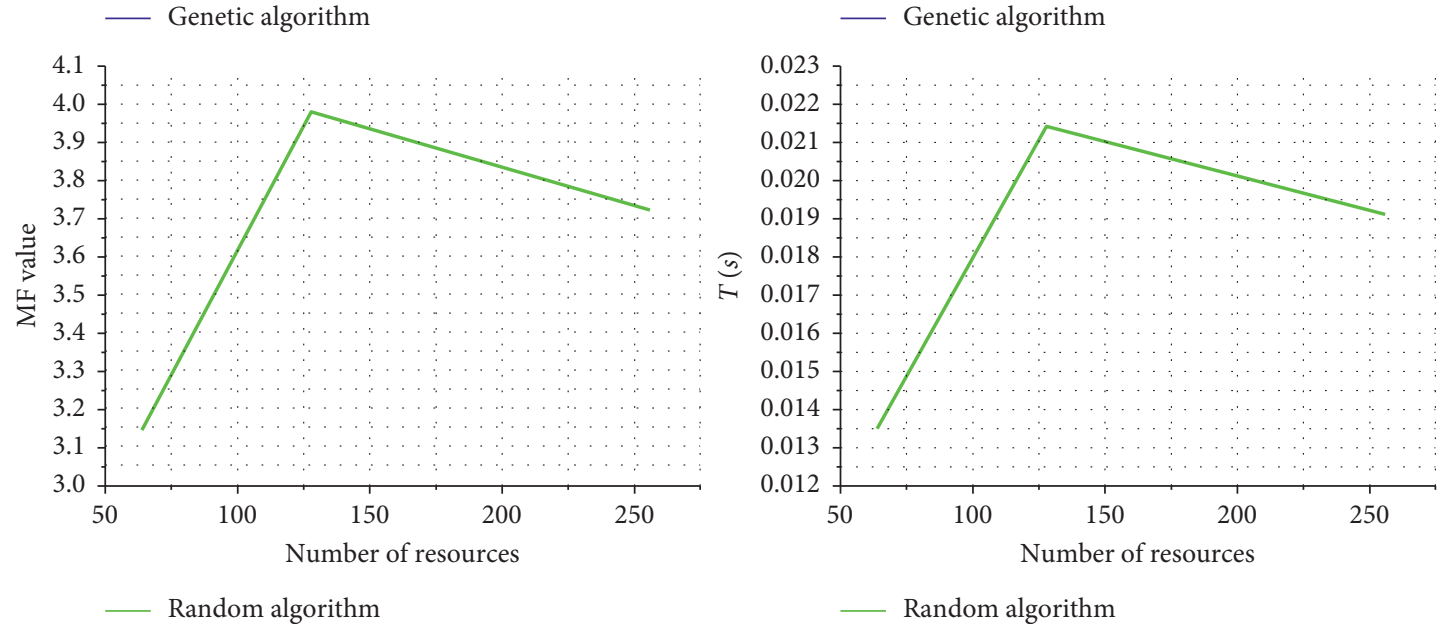

Figure 8: Average penalty function values and average running time for the three sets of samples in the two algorithm cases. 
of typical learners' learning process, it is clear that the learning effect can also be improved, and the weaknesses in learners' learning process can be found in time and improved in intensive training.

In terms of learner categories, the range of values for each feature of the typical three learners was unified, and the radar plot of the typical learner's feature distribution was obtained after the transformation, as shown in Figure 7.

Compared with the radar plots of each characteristic of each of the three categories of learners in the cluster analysis in Figure 6, the radar plots of each characteristic distribution of typical learners shown in Figure 7 are similar to Figure 6, and it can be concluded from the visualization of Figure 7 that the selected typical learner 1 belongs to category 1 , learner 2 belongs to category 2, but learner 3 does not belong to any category. This does not exclude the factor of individual phenomena. Both learner 3 and category 3 learners prefer to learn in a quiet environment; however, learner 3 has a lower reading ability, and the system recommends less difficult resources, which is exactly the opposite of category 3.

Figure 8 shows the average penalty function value (MF) and the average time to run the program (MT) for the 6 cases for 536 students. Larger penalty function values indicate a mismatch between the characteristics of the learners and the characteristics of the selected material.

From Figure 8, the range of MF values is [0.8200, 1.1893] in the case of using a genetic algorithm. This indicates that the value of the penalty function for the sequence of learning materials selected using the genetic algorithm is low and the selected material features are more compatible with the characteristics of the learners. When using the stochastic algorithm, the MF values vary in the range [4.83436, 4.9673], which indicates that the penalty function values of the sequences of learning materials selected using the stochastic algorithm are higher and the selected material features do not match the learned features. The magnitude of the MF values is affected by the type of algorithm and the number of materials under each attribute. Overall, the genetic algorithm obtains smaller penalty function values, and the stochastic algorithm obtains larger penalty function values. In the case of using the genetic algorithm, the average time variation of the selected materials ranges from [9.8995, 12.79009]. In the case of using the stochastic algorithm, the mean time variation range for picking the material is $[0.00076,0.00089]$. This indicates that the time to select materials for each learner using the stochastic algorithm was short. The time to select the appropriate material for each learner using the genetic algorithm was longer. Although the randomized algorithm took much less time to run than the genetic algorithm, the learning materials selected by the genetic algorithm were more suitable for the learners than the randomized algorithm, and the selection time was mostly under 13 seconds. This indicates that the genetic algorithm can do a good job of selecting appropriate learning materials for each learner with a small amount of time spent.

\section{Conclusion}

The data of learning records were collected and analyzed by using correlation analysis, multifactor ANOVA, regression analysis, and cluster analysis on resource recommendation and learning effect, the validity of the model, resources and learning categories, and learning process to verify the validity and rationality of the study on the adaptive recommendation of learning resources in English fragmented reading proposed in this paper. From the correlation analysis, it was concluded that there was a significant correlation between question type, reading ability, cognitive style, learning goal, and learning effectiveness and a significant correlation between difficulty reading ability, learning goal, volume, and self-assessed difficulty. From the multifactor ANOVA, it was concluded that question type, question difficulty, cognitive style, and reading ability* volume had a significant effect on learners' question positivity, and regression equation analysis was done for these four independent variables on the resource model and learner model, respectively, which in turn predicted learners' question positivity. From the clustering analysis, it was concluded that different categories of learners have different tendencies towards resources, and thus, suitable resources are recommended to them. In summary, it was concluded that the selection and range of values for question types in the resource model, reading ability, cognitive style, and learning target characteristics in the learner model were reasonable, while the setting of the range of values for topics and difficulty in the resource model and the access to the volume of learning situations in the learner model needed further study.

\section{Data Availability}

The data used to support the findings of this study are available from the corresponding author upon request.

\section{Conflicts of Interest}

The authors declare that they have no conflicts of interest.

\section{Acknowledgments}

This work was supported by Graduate Education and Teaching Reform Research Project of Wuhan Sports University: Research on Graduates' Language Output Ability Training Based on Mobile Platform, Project no. YJSJG2018002.

\section{References}

[1] A. Klašnja-Milićević, M. Ivanović, B. Vesin et al., "Enhancing e-learning systems with personalized recommendation based on collaborative tagging techniques," Applied Intelligence, vol. 48, no. 6, pp. 1519-1535, 2018.

[2] J. Xiao, M. Wang, B. Jiang, and J. Li, "A personalized recommendation system with combinational algorithm for online learning," Journal of Ambient Intelligence and $\mathrm{Hu}$ manized Computing, vol. 9, no. 3, pp. 667-677, 2018. 
[3] G. Alexandridis, A. Chrysanthi, G. E. Tsekouras, and G. Caridakis, "Personalized and content adaptive cultural heritage path recommendation: an application to the Gournia and Çatalhöyük archaeological sites," User Modeling and User-Adapted Interaction, vol. 29, no. 1, pp. 201-238, 2019.

[4] D. L. Cameron, L. Di Stefano, and A. T. Papenfuss, "Comprehensive evaluation and characterisation of short read general-purpose structural variant calling software," Nature Communications, vol. 10, no. 1, pp. 1-11, 2019.

[5] J. Kua, G. Armitage, and P. Branch, "A survey of rate adaptation techniques for dynamic adaptive streaming over HTTP," IEEE Communications Surveys \& Tutorials, vol. 19, no. 3, pp. 1842-1866, 2017.

[6] T. Jevsikova, A. Berniukevičius, and E. Kurilovas, "Application of resource description framework to personalise learning: systematic review and methodology," Informatics in Education, vol. 16, no. 1, pp. 61-82, 2017.

[7] M. Jelonek and S. Mazur, "Necessary changes, adverse effects? The institutional patterns of adaptation of economics universities to changes prompted by the reform of Poland's science and higher education system," Management Learning, vol. 51, no. 4, pp. 472-490, 2020.

[8] T. K. Rodrigues, K. Suto, H. Nishiyama et al., "Machine learning meets computation and communication control in evolving edge and cloud: challenges and future perspective," IEEE Communications Surveys \& Tutorials, vol. 22, no. 1, pp. 38-67, 2020.

[9] I. Sreeram and V. P. K. Vuppala, "HTTP flood attack detection in application layer using machine learning metrics and bio inspired bat algorithm," Applied Computing and Informatics, vol. 15, no. 1, pp. 59-66, 2019.

[10] V. Mohammadi, A. M. Rahmani, A. M. Darwesh et al., "Trustbased recommendation systems in Internet of Things: a systematic literature review," Human-centric Computing and Information Sciences, vol. 9, no. 1, pp. 1-61, 2019.

[11] C. Wei, J. Niu, and Y. Guo, "DLGNN: a double-layer graph neural network model incorporating shopping sequence information for commodity recommendation," Sensors and Materials, vol. 32, no. 12, pp. 4379-4392, 2020.

[12] Q. Zhu and M. Wang, "Team-based mobile learning supported by an intelligent system: case study of STEM students," Interactive Learning Environments, vol. 28, no. 5, pp. 543-559, 2020.

[13] V. M. A. Souza, D. M. dos Reis, A. G. Maletzke, and G. E. A. P. A. Batista, "Challenges in benchmarking stream learning algorithms with real-world data," Data Mining and Knowledge Discovery, vol. 34, no. 6, pp. 1805-1858, 2020.

[14] A. A. Kharkovskaya, E. V. Ponomarenko, and A. V. Radyuk, "Minitexts in modern educational discourse: functions and trends," Training Language and Culture, vol. 1, no. 1, pp. 66-82, 2017.

[15] H. Wang, Q. Fang, Y. Chen, L. Guan, and T. Dong, "Research on the factors influencing the reading motivation of social media users from the perspective of reading promotion in China," Libri, vol. 70, no. 4, pp. 279-290, 2020.

[16] V. Bykov, D. Mikulowski, O. Moravcik et al., "The use of the cloud-based open learning and research platform for collaboration in virtual teams," Information Technologies and Learning Tools, vol. 76, no. 2, pp. 304-320, 2020.

[17] R. Agrawal and S. Prabakaran, "Big data in digital healthcare: lessons learnt and recommendations for general practice," Heredity, vol. 124, no. 4, pp. 525-534, 2020.

[18] X. Ma, J. Liang, S. Li et al., "The design and application of intelligent learning support system based on knowledge structure," US-China Education Review, vol. 8, no. 8, pp. 313-331, 2018.

[19] F. Olivon, N. Elie, G. Grelier, F. Roussi, M. Litaudon, and D. Touboul, "MetGem software for the generation of molecular networks based on the t-SNE algorithm," Analytical Chemistry, vol. 90, no. 23, pp. 13900-13908, 2018.

[20] C. Li, Z. Zhang, W. Wei, H.-C. Chao, and X. Liu, "A possible world-based fusion estimation model for uncertain data clustering in WBNs," Sensors, vol. 21, no. 3, p. 875, 2021.

[21] W. Wang, Z. Gong, J. Ren et al., "Venue topic model-enhanced joint graph modelling for citation recommendation in scholarly big data," ACM Transactions on Asian and Low-Resource Language Information Processing (TALLIP), vol. 20, no. 1, pp. 1-15, 2021.

[22] W. Wei, Q. Ke, J. Nowak, M. Korytkowski, R. Scherer, and M. Woźniak, "Accurate and fast URL phishing detector: a convolutional neural network approach," Computer Networks, vol. 178, p. 107275, 2020. 